EXTENDED REPORT

\title{
High-resolution ultrasonography of the first metatarsal phalangeal joint in gout: a controlled study
}

\author{
Stephen A Wright, Emilio Filippucci, Claire McVeigh, Arthur Grey, Maura McCarron, Walter Grassi, \\ Gary D Wright, Allister J Taggart
}

Ann Rheum Dis 2007;66:859-864. doi: 10.1136/ard.2006.062802

See end of article for authors' affiliations

Correspondence to:

Dr S Wright, Department of

Rheumatology, Musgrave

Park Hospital, Belfast BT9

7JB, Northern Ireland, UK;

drsawright@yahoo.co.uk

Accepted 16 December 2006

Published Online First

21 December 2006

Objective: To compare high-resolution ultrasound (HRUS) with conventional radiography in the detection of
erosions in the first metatarsophalangeal joints (1st MTPJs) of patients with gout and to identify the characteristic sonographic features of gout.

Methods: HRUS examination of the 1st MTPJs of both feet was performed by two independent sonographers. The presence of joint and soft-tissue pathology was recorded. $x$ Ray examination of the feet was performed on the same day and reported by the same radiologist.

Results: 39 male patients with gout and 22 age-matched control subjects (14 with an inflammatory arthropathy and 8 disease free) were studied. The agreement on erosion between HRUS and $x$ ray was poor, $\kappa=0.229$ (non-weighted), with McNemar's test being significant $(p<0.001)$ indicating a large number of false negative $x$ rays. 22 MTPJs in patients with gout had never been subjected to a clinical attack of acute gout. In these MTPJs, there were 10 erosions detected by HRUS and 3 erosions on $x$ ray. HRUS features significantly more prevalent in the patients with gout were hard and soft tophus-like lesions $(p<0.01)$ and the double contour sign $(p<0.01)$.

Conclusions: These data show that HRUS may assist in the management of gout in two ways: first, by aiding in the diagnosis by identifying the sonographic features that may be representative of the disease, and, second, by allowing the early detection of erosive joint damage and/or tophaceous deposits even in clinically silent joints.

G out is characterised by tissue deposition of monosodium urate crystals as a result of hyperuricaemia, which can lead to acute attacks of gouty arthritis or formation of tophi (aggregated deposits of monosodium urate). ${ }^{1}$ Several British and American surveys have estimated the prevalence of gout to be 2.6-8.4 per 1000 in adults, with the prevalence increasing with age to rates of 24 per 1000 in men and 16 per 1000 in women aged $65-74$ years. $^{2}$ Gout has a predilection for the first metatarsophalangeal joint ( $1^{\text {st }}$ MTPJ), with as many as $50-70 \%$ of first gout attacks occurring here. ${ }^{3}$ The treatment of an acute attack of gout is based on a combination of nonsteroidal anti-inflammatory drugs (NSAIDs), colchicine and corticosteroids, but this does not tackle the underlying problem of hyperuricaemia. Hypouricaemic therapy is generally life long and the introduction of such treatment requires careful consideration. Evidence of radiographic bone erosions and visible tophaceous deposits are included in the current indications for considering hypouricaemic therapy, ${ }^{1}$ to ultimately prevent ongoing joint destruction and urate nephropathy. Several previous studies have demonstrated the insensitivity of conventional posteroanterior $x$ rays in depicting early bone erosions, ${ }^{45}$ and soft-tissue deposits (such as tophi) are not always visible.

Musculoskeletal high-resolution ultrasound (HRUS) is rapidly evolving into an important method for confirming the primary diagnosis and monitoring therapeutic response in many rheumatic conditions. The particular properties possessed by HRUS that lend it to these roles include a lack of ionising radiation, the ability to scan in multiple planes, high spatial resolution, real-time examination capability and relatively low operational costs. ${ }^{6}$ The production of higher frequency transducers (7.5-22 MHz) has enhanced the visualisation of more superficial structures including small-joint erosions and allows detailed assessment of soft-tissue pathology. HRUS machines with power Doppler capability further enhance the investigation by allowing quantification of inflammatory joint activity.

In other erosive arthropathies, HRUS has been shown to be more sensitive than conventional $x$ ray in detecting erosions, ${ }^{78}$ thus leading to earlier treatment to prevent further joint destruction and loss of function.

The aims of this study were: (1) to compare HRUS with conventional radiography in the detection of erosions in the $1^{\text {st }}$ MTPJs of patients with gout; (2) to identify the characteristic sonographic features of gout in the $1^{\text {st }}$ MTPJs; and (3) to relate the sonographic features to clinical features.

\section{PATIENTS AND METHODS}

\section{Subjects}

The local ethics committee at Queens University, Belfast, Northern Ireland, UK, gave approval for the study. Patients with gout were recruited from rheumatology clinics in Belfast. They either had crystal confirmation of gout after joint aspiration or were diagnosed on the basis of the American Rheumatism Association guidelines ${ }^{9}$ (box 1). Patients with arthritis other than gout were recruited from Musgrave Park Hospital, Belfast, Northern Ireland, UK, as disease controls, and healthy subjects were recruited from hospital staff as diseasefree controls.

\section{Clinical and laboratory investigation}

All subjects underwent a detailed clinical evaluation including disease history, clinical examination, laboratory testing and radiological assessment on the same day as the sonographic evaluation. Patients were specifically asked to recall whether an

Abbreviations: HRUS, high-resolution ultrasound; MTPJ metatarsophalangeal joint; NSAID, non-steroidal anti-inflammatory drug; $\mathrm{OA}$, osteoarthritis 
Box 1: American Rheumatism Association criteria for the diagnosis of gout

(1) More than one attack of acute arthritis

(2) Maximum inflammation developed within 1 day

(3) Monoarthritis attack

(4) Redness observed over joints

(5) First metatarsophalangeal joint (MTPJ) painful and swollen

(6) Unilateral first MTPJ attack

(7) Unilateral tarsal joint attack

(8) Tophus (proven or suspected)

(9) Hyperuricaemia

(10) Asymmetric swelling within a joint on $x$ ray

(11) Subcortical cyst without erosions on $x$ ray

(12) Monosodium urate monohydrate microcrystals in joint during attack

(13) Joint-fluid culture negative for organisms during attack

When criteria (12) and (13) are removed, the sensitivity for gout is $85 \%$, with a specificity of at least $93 \%$.

acute attack of gout had ever occurred in their MTPJs. Two rheumatologists-in-training independently assessed the $1^{\text {st }}$ MTPJs of both feet in each patient for signs of active inflammation and graded it as follows: 0, no tenderness; 1, mild tenderness; 2, moderate tenderness; and 3, severe tenderness. Routine laboratory testing was undertaken, including full blood count, renal function, liver function, serum urate, erythrocyte sedimentation rate and $C$ reactive protein. Standard dorsiplantar weight-bearing and lateral weight-bearing $x$ ray views of both feet were taken. The film-focus distance was $100 \mathrm{~cm}$. For the dorsiplantar view, the penetration was $50 \mathrm{kV}$ and the contrast density was $4.5 \mathrm{mAs}$, and for the lateral view, the penetration was $50 \mathrm{kV}$ and the contrast density was $8 \mathrm{mAs}$. The films were scored by an experienced musculoskeletal radiologist for the presence of erosions, joint space narrowing and visible tophi. The radiologist was blinded to the clinical and sonographic details.

\section{HRUS assessment}

All scans were performed using a Sonoline Antares (Siemens, Munich, Germany) machine with a $5-13 \mathrm{MHz}$ linear array transducer. A water-based gel was applied to the skin to provide an acoustic interface. All patients were independently scanned by two rheumatologists trained in musculoskeletal ultrasonography. One (EF) had >10 years of experience in musculoskeletal ultrasonography and the other (SAW) had recently passed a competency assessment in musculoskeletal sonography. ${ }^{10}$ Both first MTPJs were scanned in all subjects. Each joint was scanned in both longitudinal and transverse planes on both dorsal and medial sides. Power Doppler assessment of each joint was carried out with settings standardised to a pulse repetition frequency of $400-500 \mathrm{~Hz}$ and low wall filters. The power Doppler colour gain was adjusted to a level just below the disappearance of colour signs under the bony cortex as recommended by Rubin et al. ${ }^{11}$ Each sonographer was blinded to the clinical details, radiographic findings and the sonographic results of the other observer.

All patients were independently scanned by both sonographers. Each sonographer acquired a set of sonographic images that were digitally stored and used it to complete a standardised proforma off-line. The interobserver agreement between the two sonographers was calculated using the data from the standardised proforma, filled in independently by the two investigators. Data obtained by the experienced sonographer were used for the final analysis. Consensus between the sonographers was obtained before the beginning of the study on all of the sonographic findings reported in the standardised proforma (table 1) by scanning different patients not involved in the study.

The proforma was written to reflect what little evidence was available on HRUS in gout. An erosion was defined as a definite interruption in the cortical margin seen in both longitudinal and transverse views. Erosions were classified according to the bones involved (metatarsal or phalangeal), the position of the erosions (dorsal or medial) and whether they were unifocal or multifocal. Electronic callipers were used to measure the erosion, which was then categorised into a semiquantitative scale ( small erosion $=<2 \mathrm{~mm}$, moderate erosion $=2-4 \mathrm{~mm}$ and large erosion $=>4 \mathrm{~mm}$ ). If multifocal erosions were seen, the largest erosion was measured and recorded. HRUS examination was also focused on assessing both articular and peri-articular soft-tissue abnormalities. Joint inflammation was recorded when either joint effusion or synovial hypertrophy was detected. The presence of power Doppler activity was graded on a semiquantitative scale that had been agreed upon by the two sonographers before the commencement of the study by scanning different patients (not involved in this study) to come to a consensus. This scale has previously been described $^{12}(0$, no flow in the region of interest; 1 , single vessel signals; 2, confluent vessels signals in less than half the area of the region of interest; 3 , vessel signals in more than half the region of interest).

\section{Statistical analysis}

All statistical analysis was performed using SPSS V.12.1. Descriptive variables are presented as mean (SD) and compared using the $t$ test of independent samples or $t$ test of proportions, whichever was appropriate. The non-weighted $\kappa$ was used to test agreement between HRUS and $x$ ray, with McNemar's test used to determine whether significant bias occurred. The correlation between variables was determined using Spearman's correlation coefficient with Bonferroni's correction being applied where appropriate. Significance was set at $p<0.05$. The level of agreement between the two sonographers was evaluated using Cohen's- $\kappa$ (linear weights).

\section{RESULTS}

\section{Demographics}

In all, 39 patients with gout were studied; of these, 11 patients had crystal confirmation and 28 patients were diagnosed on the basis of history. There were 22 control subjects, 14 disease controls and 8 disease-free controls. Table 2 shows the demographic characteristics of the participants.

Patients with gout had significantly higher body mass index, alcohol consumption and serum urate levels. In patients with gout, mean (SD) disease duration was 12 (8) years. A total of 32 patients were on allopurinol (mean dose 300 (200) mg), 25 patients were on a NSAID or COX2 inhibitor ( 13 patients were taking NSAIDs, 12 patients were on a COX2 inhibitor) and 3 patients were on colchicine. Of the 14 disease control patients, 6 had sero-positive rheumatoid arthritis (mean disease duration 11 (4) years, 4 patients were on methotrexate and 2 patients received sulfasalazine), 4 had osteoarthritis (mean disease duration 5 (2) years), 2 had psoriatic arthritis (mean disease duration 7 (3) years, both on methotrexate) and 2 had spondyloathropathy (mean disease duration 9 (4) years, both on NSAIDs). 
Table 1 Description of high-resolution ultrasound findings used for consensus between sonographers

\begin{tabular}{ll}
\hline HRUS findings & Description \\
\hline Bone erosion & $\begin{array}{l}\text { An intra-articular break of the bone profile that is detectable in at least two } \\
\text { perpendicular planes }\end{array}$ \\
$\begin{array}{l}\text { A compressible anechoic intra-articular area } \\
\text { Joint effusion }\end{array}$ & $\begin{array}{l}\text { An uncompressible hypoechoic intra-articular area } \\
\text { Intra-articular coloured spots }\end{array}$ \\
Pownor-Doppler signal & A deposit showing an inhomogeneously echoic "echotexture" \\
Soft tophus-like lesion & A deposit appearing as a hyperechoic band generating a posterior acoustic shadow \\
Hard tophus-like lesion & A deposit showing ultrasound features of both soft and hard tophus \\
Mixed tophus-like lesion & A focal or diffuse enhancement of the superficial margin of the articular cartilage whose \\
Double contour & reflectivity is independent of the angle of insonation \\
& Spots $<1$ mm in size with the same echogenicity of the bony cortex \\
\hline
\end{tabular}

HRUS, high-resolution ultrasound.

Among the patients with rheumatoid arthritis, 2 had clinical and radiological involvement of their $1^{\text {st }}$ MTPJs. Two patients with psoriatic arthritis had clinical and radiological involvement of their $1^{\text {st }}$ MTPJs. None of the patients with spondyloarthropathy had clinical or radiological involvement of their $1^{\text {st }}$ MTPJs. Of the four patients with osteoarthritis (OA), three had knee OA and one patient had nodal OA of the hands. Patients with nodal OA had radiological evidence of OA in one of their MTPJs.

\section{Erosion in patients with gout}

A total of $781^{\text {st }}$ MTPJs in patients with gout were studied. In all, 52 (67\%) MTPJs had evidence of erosions on HRUS (fig 1). Of these joints, 15 (29\%) had a focal erosion, and 37 (71\%) had multifocal erosions. The mean size of the erosion (largest linear dimension) on HRUS was $2.4(0.8) \mathrm{mm}$, with $92 \%$ of the erosions present on the medial aspect of the metatarsal head, $7 \%$ on the dorsal aspect of the metatarsal head and $2 \%$ on the medial aspect of the phalangeal base. In all, 19 erosions had power Doppler activity visible within the erosion. On $x$ ray, 22 (28\%) MTPJs had visible erosions. The agreement, on erosion, between HRUS and $x$ ray was poor, $\kappa=0.229$ (non-weighted), with McNemar's test being significant $(\mathrm{p}<0.001)$ indicating a large number of false negative $x$ rays. Analysing the erosions present on the medial head of the metatarsal only, the agreement between HRUS and $x$ ray was still poor, $\kappa=0.34$ (non-weighted).

Erosions detected on HRUS had a significant positive correlation with disease duration $\left(\mathrm{r}_{\mathrm{s}}=0.42 ; \mathrm{p}=0.01\right)$ and $\mathrm{a}$ weaker correlation with the number of acute attacks of clinical gout in the MTPJ $\left(r_{s}=0.34 ; p=0.05\right)$. Furthermore, there was a significant correlation between the presence of erosions on HRUS and the presence of hard, soft or mixed tophus-like lesions on HRUS $\left(\mathrm{r}_{\mathrm{s}}=0.59 ; \mathrm{p}=0.01\right)$. There were 22 MTPJs that patients recalled never having been subjected to a clinical attack of acute gout. Of these joints, $10(45 \%)$ were found to have erosions by HRUS (five with a focal erosion and five with multifocal erosions, mean (SD) size $2.1(0.7) \mathrm{mm}$, all located on the medial aspect of the metatarsal head). In these 22 MTPJs, $x$ ray detected only 3 (14\%) joints with erosions. Furthermore, 7 (32\%) of these joints had HRUS evidence of tophus-like lesions and $4(18 \%)$ had evidence of the double contour sign.

\section{Comparison of HRUS findings between groups}

The interobserver agreement between the two sonographers was $\kappa=0.87$ for HRUS erosions and $\kappa=0.76$ for HRUS softtissue pathology. Table 3 shows the comparison of HRUS and $x$ ray findings between the groups.

As can be seen, there were significantly more erosions on MTPJs of patients with gout on HRUS (patients with gout had $52(66 \%)$ MTPJs with erosions compared with disease controls who had $12(43 \%)$ MTPJs with erosions, $p=0.038$ and patients with gout versus healthy controls $1(6 \%)$ MTPJ with erosion, $\mathrm{p}<0.001$ ). There was no difference in the proportion of patients with gout with synovial hypertrophy or joint effusion when compared with the disease controls, but the healthy controls had significantly less. As can be seen from table 3, in the healthy controls both erosion and soft-tissue pathology were found. This was from one patient who is currently undergoing follow-up and investigation. There were significantly more tophus-like lesions and double contours detected in the MTPJs of patients with gout than either controls groups. Soft tophuslike lesion (fig 2) was present in 27 (35\%) MTPJs of patients with gout compared with 4 (14\%) MTPJs of disease controls $(p<0.02)$ and in no MTPJs of the healthy controls $(p<0.01)$. Hard tophus-like lesion (fig 3 ) was present in 2 (3\%) MTPJs of patients with gout and in none of the MTPJs from the controls. A mixed tophaceous-like lesion pattern was present in 9 (12\%) MTPJs of patients with gout and in none of the controls, $\mathrm{p}<0.05$. The double contour sign (figs $2 \mathrm{~A}$ and 3 ) was present in $17(22 \%)$ MTPJs of gout patients and in none of the controls, $\mathrm{p}<0.01$.

Table 2 Demographic characteristics showing significantly higher body mass index, alcohol consumption and serum urate levels in patients with gout

\begin{tabular}{llll}
\hline & Patients with gout $(\mathbf{n}=39)$ & Control subjects $(\mathbf{n}=22)$ & $\mathbf{p}$ Value \\
\hline Male (\%) & 100 & 86 & $\mathrm{NS}$ \\
Age (years) & $52(11)$ & $53(16)$ & $\mathrm{NS}$ \\
BMI $\left(\mathrm{kg} / \mathrm{m}^{2}\right)$ & $30(4)$ & $26(6)$ & 0.007 \\
Alcohol (units/week) & $13(12)$ & $6(10)$ & 0.027 \\
Urate (mmol/l)* & $0.41(0.11)$ & $0.30(0.08)$ & 0.002 \\
\hline
\end{tabular}

BMI, body mass index; NS, non-significant.

All results are expressed as mean (SD) unless indicated otherwise.

*Normal laboratory range is $0.23-0.46 \mathrm{mmol} / \mathrm{l}$ for males, and $0.19-0.36 \mathrm{mmol} / \mathrm{l}$ for females. 

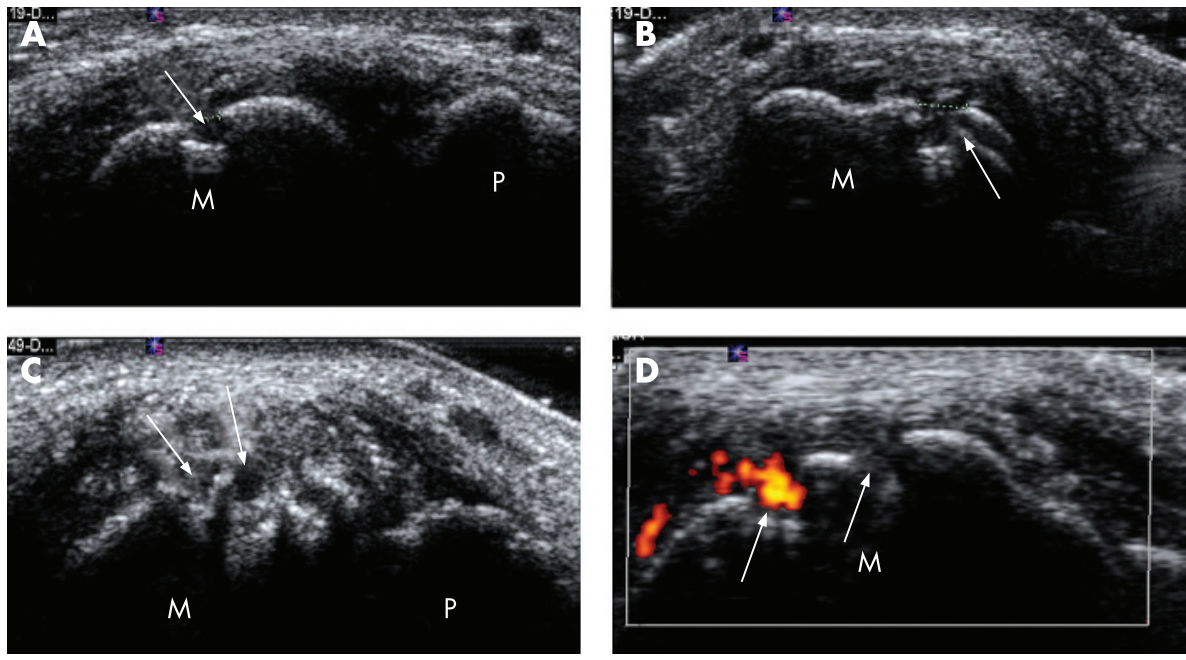

Figure 1 High-resolution ultrasound (HRUS) representation of erosions in metatarsophalangeal joints (MTPJs) of patients with gout. (A) Longitudinal and (B) transverse HRUS of right $1^{\text {th }}$ MTPJ showing a focal bone erosion on the metatarsal head. (C) Longitudinal HRUS of left $1^{\text {st }}$ MTPJ showing multifocal erosions on the metatarsal head. (D) Transverse HRUS with power Doppler of right $1^{\text {st }}$ MTPJ showing two erosions on the metatarsal head, one with power Doppler signal (hot erosion) and the other with no power Doppler signal (cold erosion). $M$, metatarsal; $P$, phalanx; white arrow, erosion.

\section{Comparison between HRUS and clinical findings}

Clinical examination of the MTPJs revealed that 39 (50\%) joints had no tenderness, 27 (35\%) joints had mild, 7 (9\%) joints had moderate and $5(6 \%)$ joints had marked tenderness. There was no significant correlation between clinical examination of the MTPJs and the presence of erosion on HRUS (right MTPJ $\mathrm{r}_{\mathrm{s}}=0.22 ; \mathrm{p}=0.18$ and left MTPJ $\mathrm{r}_{\mathrm{s}}=0.36 ; \mathrm{p}=0.12$ ) or the presence of joint effusion or synovial hypertrophy on HRUS (right MTPJ $\mathrm{r}_{\mathrm{s}}=0.14 ; \mathrm{p}=0.75$ and left MTPJ $\mathrm{r}_{\mathrm{s}}=0.19$; $\mathrm{p}=0.25)$.

\section{DISCUSSION}

This is the first study to demonstrate that HRUS detects significantly more erosions than $x$ ray in the $1^{\text {st }}$ MTPJs of patients with gout, and that pathological findings can occur in clinically silent joints. It also describes sonographic findings in the $1^{\text {st }}$ MTPJs that may represent changes of gout.

Previous reports, in other inflammatory joint diseases, have also demonstrated the ability of HRUS to detect more erosions than conventional $x$ ray. ${ }^{713}$ The high spatial resolution and multiplanar capability of the technique make it particularly suitable for this purpose. Conventional $x$ ray will only detect erosions that are in a plane that is tangential to the radiographic beam. Variations in film projection and penetration may further limit the quality of the $x$ ray image, which ultimately relies upon the projection of a three dimensional image on to a two dimensional medium. ${ }^{14}$ Only the standard posteroanterior and lateral $x$ rays of the MTPJs were used in this study. This may have led to underestimation of the ability of plain radiography to detect erosions, but this is an accurate reflection of everyday clinical practice.

\begin{tabular}{|c|c|c|c|}
\hline & $\begin{array}{l}\text { Patients with gout } \\
\text { ( } \mathrm{n}=78 \mathrm{I}^{\mathrm{st}} \text { MTPJs) }\end{array}$ & $\begin{array}{l}\text { Disease control } \\
\text { ( } \mathrm{n}=28 \quad 1^{\text {st }} \text { MTPJs) }\end{array}$ & $\begin{array}{l}\text { Healthy control } \\
\text { ( } n=161^{\text {st }} \text { MTPJs) }\end{array}$ \\
\hline \multicolumn{4}{|l|}{$x$ Ray } \\
\hline Erosion & $22(28)$ & $4(14)^{*}$ & 0 \\
\hline Tophi & $3(4)$ & 0 & 0 \\
\hline \multicolumn{4}{|l|}{ HRUS erosions } \\
\hline Total & $52(66)$ & $12(43)^{*}$ & $1(6) \dagger$ \\
\hline Focal & $15(19)$ & & $1(6)^{*}$ \\
\hline Multifocal & 37 (47) & $12(43)$ & 0 \\
\hline Joint effusion & $55(71)$ & $18(64)$ & $2(13) \dagger$ \\
\hline Synovial hypertrophy & $68(87)$ & $18(64)$ & $1(6) \dagger$ \\
\hline \multicolumn{4}{|l|}{ Power Doppler } \\
\hline None & $44(56)$ & $14(50)$ & $15(94) \dagger$ \\
\hline Mild & $12(15)$ & $5(18)$ & $1(6)$ \\
\hline Moderate & $14(18)$ & $4(16)$ & 0 \\
\hline Marked & $8(10)$ & $4(16)$ & 0 \\
\hline \multicolumn{4}{|l|}{ Tophus-like lesions } \\
\hline Hard & $2(3)$ & 0 & 0 \\
\hline Soft & $27(35)$ & $4(14)^{*}$ & 0 \\
\hline Mixed & $9(12)$ & 0 & 0 \\
\hline Double contour & $17(22)$ & 0 & 0 \\
\hline Hyperechoic spots & $9(12)$ & $4(14)$ & $2(13)$ \\
\hline \multicolumn{4}{|c|}{$\begin{array}{l}\text { HRUS, high-resolution ultrasound; MTPJ, metatarsophalangeal joint. } \\
\text { Results are expressed as number }(\%) \text { of MTPJs. } \\
\text { Test of proportions. * } p<0.05 \text {. and } \dagger p<0.01 \text { compared with patients having gout. } \\
\text { The proportion of MTPJs in patients with gout showing HRUS evidence of erosions, tophus-like lesions and double contour } \\
\text { was significantly greater than in the disease or healthy control groups. }\end{array}$} \\
\hline
\end{tabular}



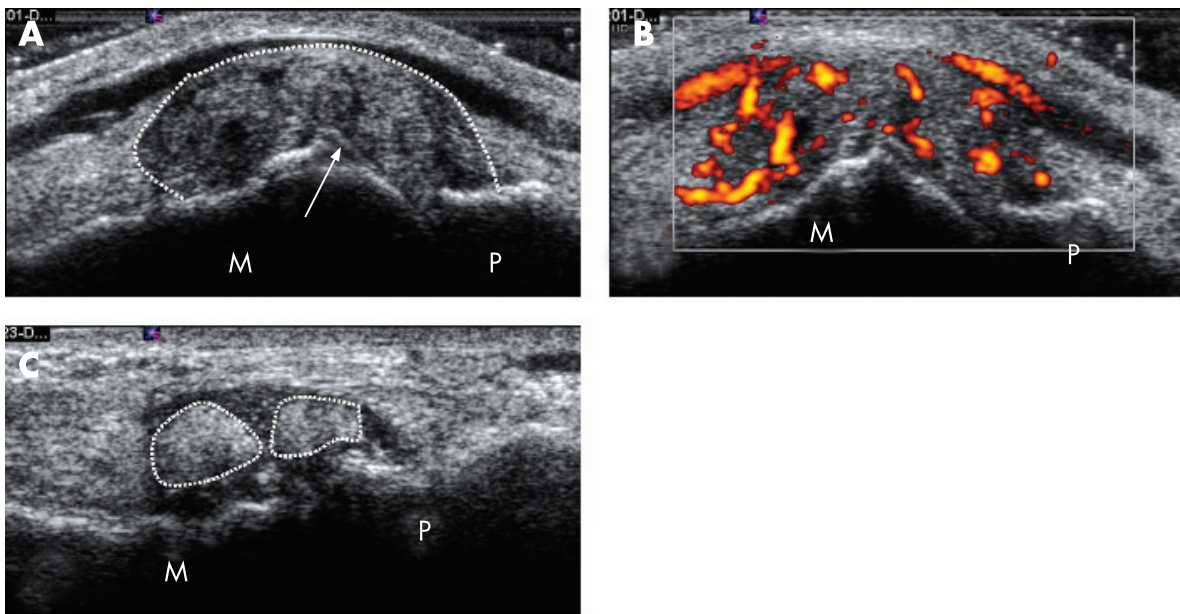

Some studies have used MRI as the "gold standard" for imaging small joints. ${ }^{7}{ }^{15}$ MRI is becoming more widely available for this purpose, but it does have certain limitations when used to detect small bony erosions. MRI cannot visualise the bone cortex directly and if marrow oedema is present, a volumeaveraging effect can lead to difficulty in interpretation. Sonography has superior spatial resolution to MRI and some or all of these factors may explain some reports showing that HRUS is superior to MRI in the detection of smaller erosions in both hands and feet. ${ }^{815}$ We did not use MRI, as the routine use of MRI for imaging of MTPJs in patients with chronic gout is rare because of the expense and time required. Others would suggest that CT should be the "gold standard" when detecting small-joint bone erosions. Although there has been no direct comparison between HRUS and CT in the detection of smalljoint bone erosions, HRUS has been shown to be superior to CT in the detection of erosions on larger bones. ${ }^{16}$ Nevertheless, further studies should be conducted using MRI or CT to confirm our findings. One disadvantage of HRUS is the inability to fully blind the sonographer to the diagnosis, as visual clues from the gross presentation and levels of joint tenderness can be elicited when placing the probe on the joint.

There are very few reports on the sonographic features of gout in both joints and soft tissues. Filippucci $e t a l^{17}$ showed the potential benefit of HRUS in the monitoring of patients with gout, whereas others have described case reports of gouty tenosynovitis, rotator cuff lesions, upper-limb cellulitis and tendon tophi. ${ }^{18-21}$ Gerster $e t$ al ${ }^{22}$ looked at the knee joint in four

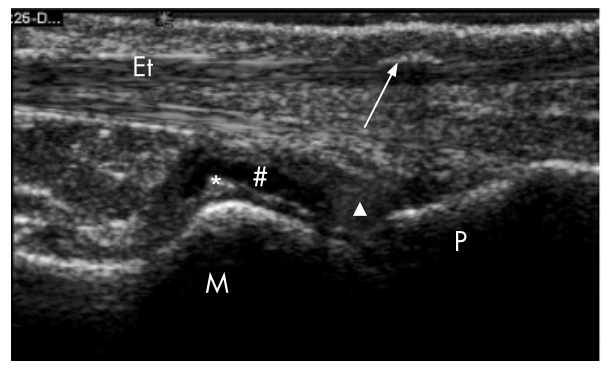

Figure 3 High-resolution ultrasound (HRUS) representation of hard tophus-like lesions in metatarsophalangeal joints (MTPJ) of patients with gout. Longitudinal HRUS of right $1^{\text {st }}$ MTPJ showing hard tophus-like lesion on extensor tendon, joint effusion, synovial hypertrophy and the double contour sign. $M$, metatarsal; $P$, phalanx; white arrow, hard tophus-like lesion; $\mathrm{Et}$, extensor tendon; ${ }^{*}$, double contour sign; \#, joint effusion; white triangle, synovial hypertrophy. patients with gout and compared CT, MRI and sonography for imaging tophaceous deposits. They concluded that CT was the best modality for imaging gouty tophi.

In the current literature, there are very few reports concerning the differences found on HRUS in different arthritides. ${ }^{23}$ In our study, there were non-specific pathological findings (eg, bone erosions, joint effusion, synovial hypertrophy and hyperechoic spots). However, sonographic findings suggesting the presence of tophus or double contour were significantly more frequent in patients with gout than in either of the control groups. The presence of these sonographic findings in the $1^{\text {st }}$ MTPJs may represent changes due to gout and could be a useful clinical tool in differentiating gout from other inflammatory arthritides. However, further studies are needed to ascertain the pathology underlying these sonographic findings.

The decision to introduce hypouricaemic therapy for chronic gout usually depends upon the frequency of gouty attacks as well as the presence of radiographic erosions and/or tophaceous deposits. Generally, it is accepted that hypouricaemic therapy should be life long with the goal of reducing and eventually eliminating gouty attacks, thereby stopping further joint destruction..$^{24}$ As expected, we demonstrated a positive correlation between the number of attacks of gout in the $1^{\text {st }}$ MTPJ and the presence of erosions, but the association between the two was weak. This indicates that other factors, other than clinical attacks, must be important predictors of joint damage. This conclusion is supported by the fact that we found evidence of erosive joint damage in MTPJs that patients recalled never having been subjected to an attack of gouty arthritis. These sonographic findings in clinically silent joints have parallels with a recent study of renal tract sonography in urate nephropathy. This showed a higher than expected incidence of urolithiasis in asymptomatic patients with gout when sonography was compared with clinical signs and symptoms alone. ${ }^{25}$ In our study, there could be a bias towards patients not recalling an attack of gout in the $1^{\text {st }}$ MTPJ. However, owing to the extreme pain and discomfort often associated with acute gout in the $1^{\text {st }}$ MTPJ, inaccurate patient recall would be minimal.

Tophaceous deposits become clinically apparent only after a long period of asymptomatic hyperuricaemia. ${ }^{24}$ If one could detect tophi at a much earlier stage of the disease, this could lead to a more timely therapeutic intervention and the prevention of joint and other organ damage. $x$ Rays cannot be relied upon for the early detection of urate deposits in soft tissues, whereas HRUS can quickly (approximately $5 \mathrm{~min}$ per MTPJ in this study) and accurately detect soft-tissue 
pathology. ${ }^{26}$ Furthermore, in our patients with gout, there was a high prevalence of sonographic features suggestive of intraarticular tophi, as well as enhancement of the superficial margin of the articular cartilage (the double contour sign). These findings were infrequent in both control groups. Four disease controls appeared to exhibit the sonographic features of soft tophus-like deposition, but this may reflect the propensity for soft tophi to be confused with extensive synovial hypertrophy.

The introduction of biological treatments for rheumatoid arthritis and other inflammatory arthritides ${ }^{27}$ has placed an obligation on rheumatologists to detect these diseases early in order to reduce joint damage by aggressive and more targeted treatment. ${ }^{28}$ To this end, the use of HRUS by rheumatologists is increasing rapidly to aid with early detection of synovitis and erosions in these patients. The same paradigm should be applied to the management of gout. New treatments to aid with uric acid suppression are currently being developed, ${ }^{29}{ }^{30}$ and the ability to detect joint damage and tophaceous deposition at an early stage using HRUS should allow earlier and more timely intervention.

HRUS may assist with the management of gout in two ways: first, by aiding in the diagnosis by identifying sonographic features that may be representative of the disease, and, second, by allowing the early detection of erosive joint damage and/or tophaceous deposits even in clinically silent joints. Both require further studies.

\section{Authors' affiliations}

Stephen A Wright, Claire McVeigh, Maura McCarron, Gary D Wright, Allister J Taggart, Department of Rheumatology, Musgrave Park Hospital, Belfast, Northern Ireland, UK

Emilio Filippucci, Walter Grassi, Cattedra di Reumatologia, Università Politecnica delle Marche, Ospedale "A Murri", Jesi (Ancona), Italy Arthur Grey, Department of Radiology, Musgrave Park Hospital, Stockmans Lane, Belfast, Northern Ireland, UK

Competing interests: None declared.

\section{REFERENCES}

1 Agudelo CA, Wise CM. Gout: diagnosis, pathogenesis, and clinical manifestations. Curr Opin Rheumatol 2001;13:234-9.

2 Lawrence RC, Helmick CG, Arnett FC, Deyo RA, Felson DT, Giannini EH, et al. Estimates of the prevalence of arthritis and selected musculoskeletal disorders in the United States. Arthritis Rheum 1998;41:778-99.

3 Grahame R, Scott JT. Clinical survey of 354 patients with gout. Ann Rheum Dis 1970;29:461-8

4 Doi K, Genant HK, Rossmann K. Comparison of image quality obtained with optical and radiographic magnification techniques in fine-detail skeletal radiography: effect of object thickness. Radiology 1976;118:189-95.

5 Hartley RM, Liang MH, Weissman BN, Sosman JL, Katz R, Charlton, JR, et al. The value of conventional views and radiographic magnification in evaluating early rheumatoid arthritis. Arthritis Rheum 1984;27:744-51.

6 Kane D, Balint PV, Sturrock R, Grassi W. Musculoskeletal ultrasound-a state of the art review in rheumatology. Part 1: current controversies and issues in the development of musculoskeletal ultrasound in rheumatology. Rheumatology (Oxford), 2004;43:823-8.
7 Wakefield RJ, Gibbon WW, Conaghan PG, O'Connor P, McGonagle D, Pease $C$, et al. The value of sonography in the detection of bone erosions in patients with rheumatoid arthritis: a comparison with conventional radiography. Arthritis Rheum 2000:43:2762-70.

8 Magnani M, Salizzoni E, Mule R, Fusconi M, Meliconi R, Galletti. Ultrasonography detection of early bone erosions in the metacarpophalangeal joints of patients with rheumatoid arthritis. Clin Exp Rheumatol 2004;22:743-8.

9 Wallace SL, Robinson H, Masi AT, Decker JL, McCarty DJ, Yu TF. Preliminary criteria for the classification of the acute arthritis of primary gout. Arthritis Rheum 1977;20:895-900.

10 Taggart A, Filippucci E, Wright G, Bell A, Cairns A, Meenagh G, et al. Musculoskeletal ultrasound training in rheumatology: the Belfast experience. Rheumatology (Oxford), 2006;45:102-5.

11 Rubin JM, Adler RS, Fowlkes JB, Spratt S, Pallister JE, Chen JF, et al. Fractional moving blood volume: estimation with power Doppler US. Radiology 1995; 197:183-90.

12 Szkudlarek M, Court-Payen M, Jacobsen S, Klardlund M, Thomsen HS, Ostergaard $M$, et al. Interobserver agreement in ultrasonography of the finger and toe joints in rheumatoid arthritis. Arthritis Rheum 2003;48:955-62.

13 Grassi W, Filippucci E, Farina A, Sala Y, Cervini C. Ultrasonography in the evaluation of bone erosions. Ann Rheum Dis 2001;60:98-103.

14 Brower AC. Use of the radiograph to measure the course of rheumatoid arthritis. The gold standard versus fool's gold. Arthritis Rheum 1990;33:316-24.

15 Szkudlarek M, Klarlund M, Narvestad E, Court-Payen M, Thomsen HS, Strandberg $C$, et al. Ultrasonography of the metacarpophalangeal and proximal interphalangeal joints in rheumatoid arthritis: a comparison with magnetic resonance imaging, conventional radiography and clinical examination. Arthritis Res Ther 2006;8:R52.

16 Alasaarela E, Suramo I, Tervonen O, Lahde S, Takalo P, Hakala M. Evaluation of humeral head erosions in rheumatoid arthritis: a comparison of ultrasonography, magnetic resonance, computed tomography and plain radiography Br J Rheumatol 1998;37:1152-6.

17 Filippucci E, Ciapetti A, Grassi W. Sonographic monitoring of gout. Reumatismo 2003;55:184-6.

18 Aslam N, Lo S, McNab I. Gouty flexor tenosynovitis mimicking infection: a case report emphasising the value of ultrasound in diagnosis. Acta Orthop Belg 2004;70:368-70.

19 Bond JR, Sim FH, Sundaram M. Radiologic case study. Gouty tophus involving the distal quadriceps tendon. Orthopedics 2004;27:18, 90-92.

20 O'leary ST, Goldberg JA, Walsh WR. Tophaceous gout of the rotator cuff: a case report. J Shoulder Elbow Surg 2003;12:200-1.

21 Wakefield RJ, Emery P, Pease C. Gout related upper limb cellulitis: an ultrasound study. J Rheumatol 2003;30:417-19

22 Gerster JC, Landry M, Dufresne L, Meuly JY. Imaging of tophaceous gout: computed tomography provides specific images compared with magnetic resonance imaging and ultrasonography. Ann Rheum Dis 2002;61:52-4.

23 Ostergaard M, Wiell C. Ultrasonography in rheumatoid arthritis: a very promising method still needing more validation. Curr Opin Rheumatol 2004; 16:223-30

24 Terkeltaub RA. Clinical practice. Gout. N Engl J Med 2003;349:1647-55.

25 Alvarez-Nemegyei J, Medina-Escobedo M, Villanueva-Jorge S, VasquesMellado J. Prevalence and risk factors for urolithiasis in primary gout: is a reappraisal needed? J Rheumatol 2005;32:2189-91.

26 Kane D, Grassi W, Sturrock R, Balint PV. Musculoskeletal ultrasound-a state of the art review in rheumatology. Part 2: Clinical indications for musculoskeletal ultrasound in rheumatology. Rheumatology (Oxford) 2004;43:829-38.

27 Shanahan JC, Moreland LW, Carter RH. Upcoming biologic agents for the treatment of rheumatic diseases. Curr Opin Rheumatol 2003;15:226-36.

28 Vries-Bouwstra JK, Dijkmans BA, Breedveld FC. Biologics in early rheumatoid arthritis. Rheum Dis Clin North Am 2005;31:745-62.

29 Becker MA, Schumacher HR Jr, Wortmann RL, MacDonald PA, Eustace D, Palo WA, et al. Febuxostat compared with allopurinol in patients with hyperuricaemia and gout. N Engl J Med 2005;353:2450-61.

30 Ganson NJ, Kelly SJ, Scarlett E, Sundy JS, Hershfield MS. Control of hyperuricaemia in patients with refractory gout, and induction of antibody against poly(ethylene) glycol (PEG), in a phase I trial of subcutaneous PEGylated urate-oxidase. Arthritis Res Ther 2005;8:R12. 